\title{
A HUGE PARASITIC FIBROID CAUSING HERNIA
}

\author{
Geetha $\mathrm{K}^{1}$, Jamila Hameed ${ }^{2}$, Seethalakshmi³ ${ }^{3}$ Grace $^{4}$, Madhumitha ${ }^{5}$
}

\section{HOW TO CITE THIS ARTICLE:}

Geetha K, Jamila Hameed, Seethalakshmi, Grace, Madhumitha. "A Huge Parasitic Fibroid Causing Hernia". Journal of Evolution of Medical and Dental Sciences 2014; Vol. 3, Issue 28, July 14; Page: 7807-7810, DOI: $10.14260 /$ jemds/2014/2976

ABSTRACT: Leiomyoma is the most common estrogen dependent benign tumour of the uterus occurring in the reproductive age. It is composed of smooth muscle and fibrous tissue. Asymptomatic myomas can be present in $50 \%$ of cases. It causes mainly menstrual problems like menorrhagia, metorrhagia, dysmenorrhoea and also infertility. Nowadays it is very rare to get huge myomas because most of the patients for any vague, trivial complaints undergoes scan, which picks up even very small myomas. We are presenting a very huge fibroid causing hernia in a patient 48years old P3L3 who developed hernia, following puerperal sterilization done. She had a hernia repair done 20 years ago. Now she developed recurrent hernia due to the huge abdominal mass $22 \times 22 \mathrm{cms}$ of a parasitic fibroid. She was taken up for laporotomy. The mass was removed in addition total hysterectomy with bilateral salpingo oophorectomy was done. This case is presented here because of its rarity and its management was quite tough- a Herculean task of course.

KEYWORDS: Hernia, parasitic fibroid, Subserous fibroid, total abdominal hysterectomy with salpingo-oophorectomy.

CASE REPORT: A 48years old lady P3L3 got admitted with the history of menorrhagia, abdominal pain and breathlessness since 6months. On examination she was found to be anemic. Uterus was palpable up to 24 weeks size and she had a separate huge mass $22 \times 22 \mathrm{cms}$ size extending up to the umbilicus and the right lumbar region. She had a scar in the infra umbilical region with incisional hernia. Cervix was healthy.

No significant medical history present. She was transfused with two units of blood. Other blood investigations were normal. X-Ray chest showed cardiomegaly with left ventricular hypertrophy. Intra venous pyelogram showed bilateral hydroureter. Ultrasound showed umbilical hernia, uterus had multiple fibroids-sizes: $20 \times 17 \times 10 \mathrm{cms}$ and a huge fundal fibroid of size $22 \times 22 \mathrm{cms}$. The right ovary was cystic. Computed tomography showed large heterogeneous multi loculated abdomino pelvic mass of size $22 \times 20 \mathrm{cms}$ with internal degeneration and calcifications from the pelvis.

MANAGEMENT: Patient was taken up for laporotomy. There were plenty of adhesions which were released. A huge subserous fundal fibroid, $24 \times 24 \mathrm{cms}$ with a twisted pedicle almost getting detached was attached to the Fundus of the uterus on the right side. There were plenty of vessels from the omentum feeding the fibroid. The whole omentum was attached to the surface of the fibroid. It was very vascular. The clamps were applied on the twisted pedicle of the subserous fibroid as well as on the huge vessels which were feeding it.

Thanks to the general surgeon, the task was made easy. Part of the omentum had to be sacrificed because it was adherent to the surface of the subserous fibroid. The uterus was enlarged to 24 weeks size which had intramural fibroids. Total abdominal hysterectomy with bilateral salpingo oophorectomy was done. Hernia repair was done meticulously with mesh. 
The specimen of subserous fibroid (parasitic fibroid) was sent for histopathology (HPE). The uterus with the fibroid, tubes, ovaries and the excised omentum were also sent for HPE. The weight of the specimen was $4.6 \mathrm{kgs}$. The histopathology report showed simple hyperplasia of the endometrium and multiple leiomyoma. The omentum had inflammatory changes.

DISCUSSION: Most of the Leiomyomas are asymptomatic. Some fibroids cause pressure symptoms, infertility, menorrhagia, ${ }^{1}$ pain and breathlessness. Rapid growth of a fibroid suggests its tendency for malignancy ${ }^{2}$ and these fibroids should be removed as early as possible when they cause symptoms. Gonadotropin releasing hormones agonists are suggested in asymptomatic, perimenopausal age group women for shrinkage of myoma. ${ }^{3}$ In case of very huge myomas, they are not very helpful. If the uterine size is more than 12 weeks, regardless of symptoms in addition to the location, surgical removal is mandatory. ${ }^{4}$

Intravenous pyelogram showed bilateral hydroureter, for which bilateral D-J stenting must be done prior to the surgery.5,6 When pain is associated with leiomyoma, it is most probably due to degeneration, twisted pedicle, malignancy, rapid growth and other associated pelvic pathology.

The incidence of sarcomatous degeneration is $0.7 \%$. Leiomyoma have monoclonal origin, arise from benign cases or may develop de nova. ${ }^{7}$ To consider a leiomyoma as malignant-the mitotic figures should be greater than 15/10 High Power Field, ${ }^{8}$ Tumour cell necrosis, nuclear atypia and pleomorphism are noted.

CONCLUSION: It has become a rare entity to see such huge fibroids with disfigurement. Most of the patients seek the help of gynaecologists even for small hypoechoic areas seen in the routine ultrasound investigations. In the perimenopausal age most of the women from rural side think the cause of menorrhagia to be "normal" because she is on the verge of reaching menopause. Even the abdominal distension causing difficulty in walking was mistaken for "gas".

Such a huge parasitic fibroid causing hernia was mistaken for abdominal fat according to the patient. The need of the hour is a proper advice for the women who are illiterate and ignorant. They should be properly advised, investigated and managed. When the lady was questioned about the huge fibroid, she could only give answers telling that her abdomen got distended because of bearing children. The periodic camps should be conducted and the message should be passed from one lady to other.

\section{REFERENCES:}

1. Wegienka G, etal. Self-reported heavy bleeding associated with uterine leiomyomata. Obstet Gynecol. 2003 Mar; 101 (3): 431-7.

2. Parker WH, Fu YS, Berek JS. Uterine sarcoma in patients operated on for presumed leiomyoma and rapidly growing leiomyoma. Obstet Gynecol. 1994 Mar; 83 (3): 414-8.

3. Nakamura Y, Yoshimura Y. Treatment of uterine leiomyomas in perimenopausal women with gonadotropin-releasing hormone agonists. Clin Obstet Gynecol. 1993 Sep; 36 (3): 660-7.

4. Friedman AJ, Haas ST. Should uterine size is an indication for surgical intervention in women with myomas? Am J Obstet Gynecol. 1993 Mar; 168 (3 Pt 1): 751-5.

5. Aungst MJ, Sears CL, Fischer JR. Ureteral stents and retrograde studies: a primer for the gynaecologist. Curr Opin Obstet Gynecol. 2009 Oct; 21 (5): 434-41. 


\section{CASE REPORT}

6. Redan JA, McCarus SD. Protect the ureters. JSLS. 2009 Apr-Jun; 13 (2): 139-41.

7. Zhang $\mathrm{P}$ etal. Use of $\mathrm{X}$-chromosome inactivation pattern to determine the clonal origins of uterine leiomyoma and leiomyosarcoma. Hum Pathol. 2006 Oct; 37 (10): 1350-6.

8. Hendrickson MR etal. Atypical leiomyomas of the uterus: a clinicopathologic study of 51 cases. Am J Surg Pathol. 2013 May; 37 (5): 643-9.
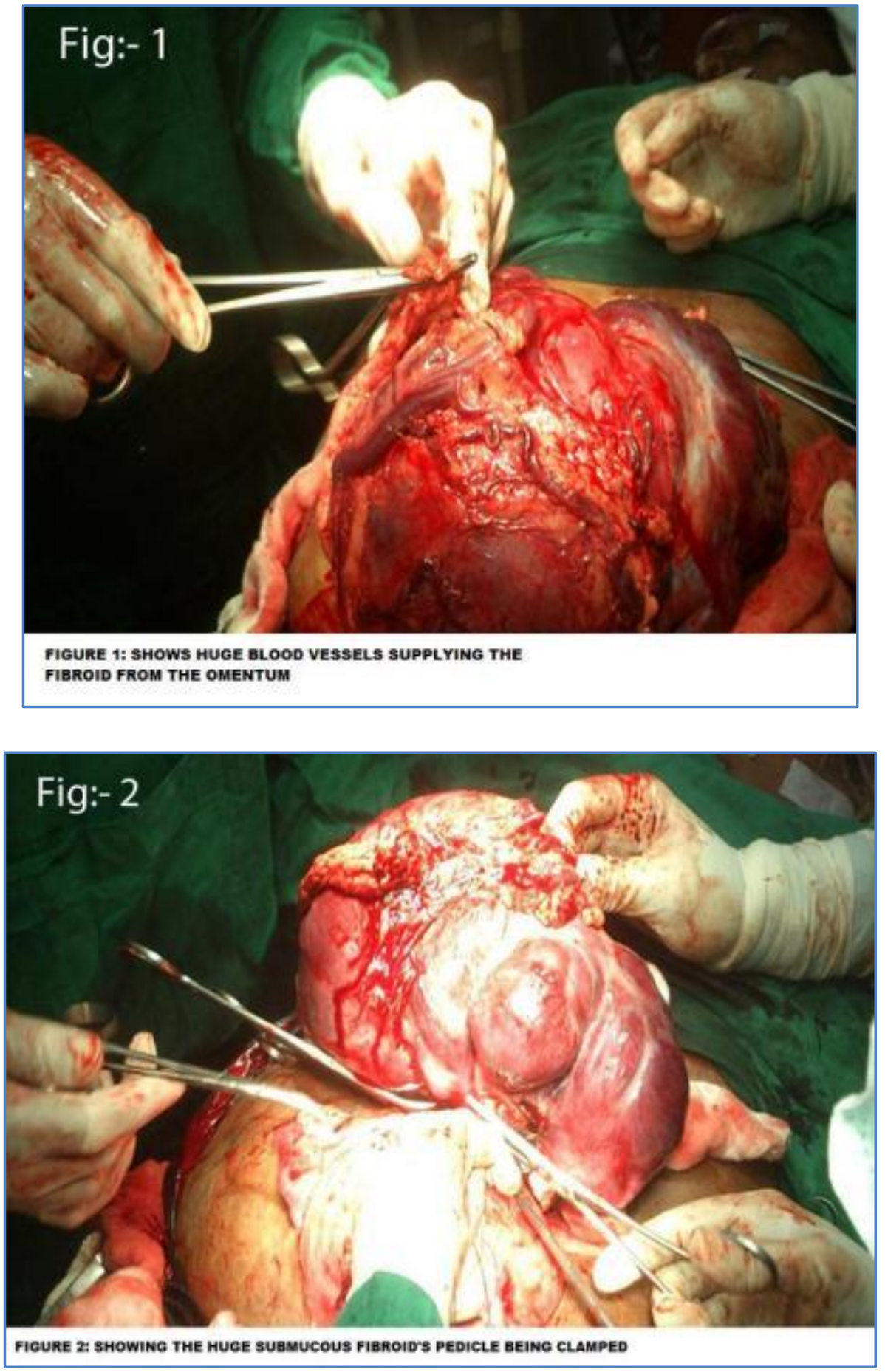


\section{CASE REPORT}

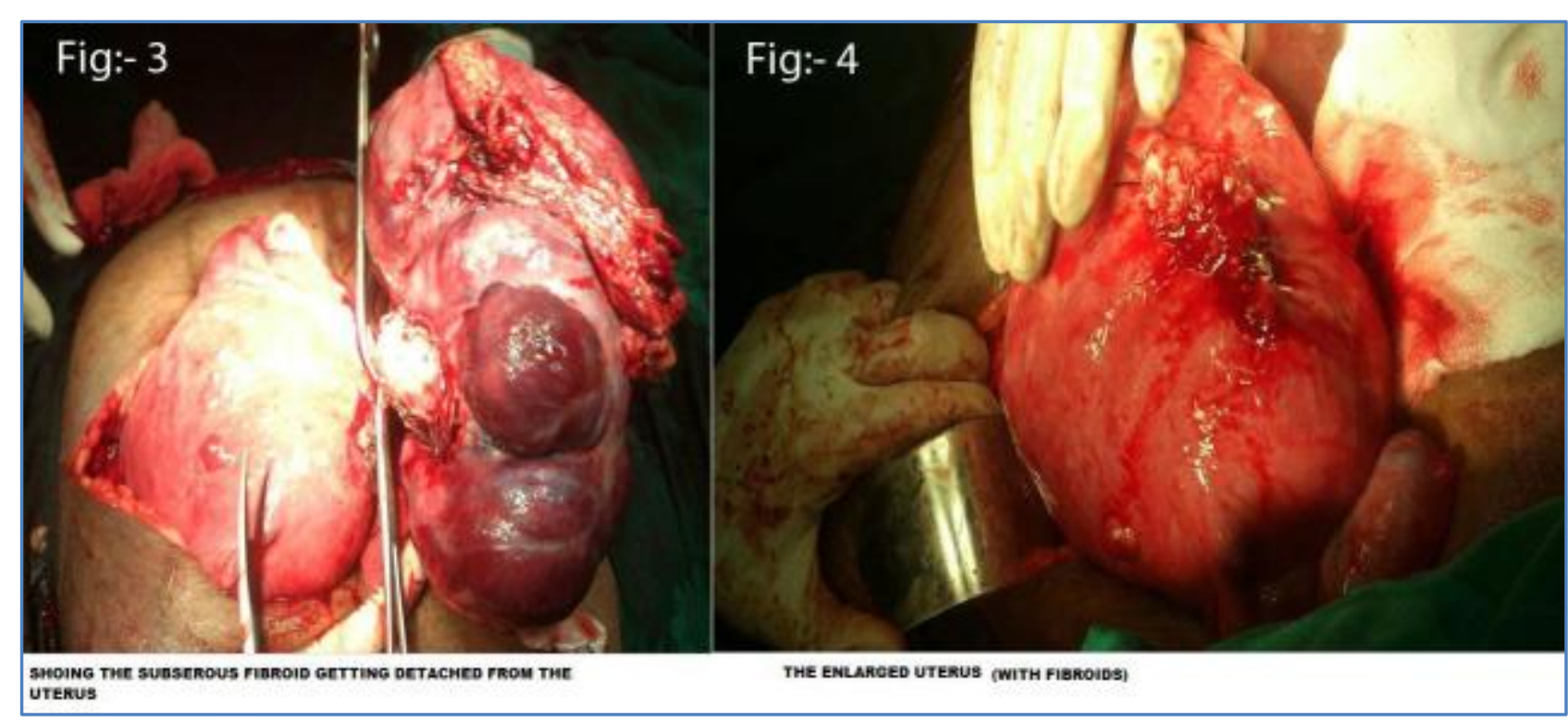

\section{AUTHORS:}

1. Geetha K.

2. Jamila Hameed

3. Seethalakshmi

4. Grace

5. Madhumitha

\section{PARTICULARS OF CONTRIBUTORS:}

1. Assistant Professor, Department of Obstetrics and Gynaecology, Vinayaka Mission's Medical College and Hospitals, Karaikal.

2. Professor, Department of Obstetrics and Gynaecology, Vinayaka Mission's Medical College and Hospitals, Karaikal.

3. Tutor, Department of Obstetrics and Gynaecology, Vinayaka Mission's Medical College and Hospitals, Karaikal.

4. Post Graduate, Department of Obstetrics and Gynaecology, Vinayaka Mission's Medical College and Hospitals, Karaikal.
5. Post Graduate, Department of Obstetrics and Gynaecology, Vinayaka Mission's Medical College and Hospitals, Karaikal.

\section{NAME ADDRESS EMAIL ID OF THE CORRESPONDING AUTHOR:}

Dr. Jamila Hameed,

Professor,

Department of Obstetrics \& Gynaecology,

Vinayaka Mission's Medical College \& Hospitals,

Karaikal- 609609.

E-mail: jamilahameed@gmail.com

Date of Submission: 11/04/2014.

Date of Peer Review: 12/04/2014.

Date of Acceptance: 30/04/2014.

Date of Publishing: 11/07/2014. 